\title{
HOMENAJE AL DR. JORGE ENRIQUE SCHNEIDER, CON MOTIVO DE CELEBRARSE EL CINCUENTENARIO DE LA FUNDACIÓN DEL LICEO DE APLICACIÓN DE SANTLAGO
}

SANTIAGO: INSTITUTO CULTURAL

GERMANO-CHILENO, 1942.

(Fragmento) 


\title{
LA INFLUENCIA DE DON JORGE ENRIQUE SCHNEIDER EN LA ENSEÑANZA SECUNDARIA Y EN EL LICEO DE APLICACIÓN.
}

\author{
Por Santiago Peña y Lillo.
}

En el desarrollo de la Enseñanza Secundaria de nuestro país, existe un acontecimiento de tal magnitud, que por sí sólo ha sido capaz de señalarle rumbos a esta rama de la Educación, en su marcha ascendente hasta nuestros días. Ese hecho es la apertura del Instituto Pedagógico y, formando su obra complementaria, el nacimiento del Liceo de Aplicación donde debían ponerse en práctica nuevos principios didácticos que la Universidad comenzaba, con clara visión a impartir, ya desde la última década del siglo pasado.

Se trataba, en primer lugar, de crear una prestigiosa carrera, basada en altos estudios de carácter científico; en seguida, de preparar a los maestros secundarios en el conocimiento de ciertas técnicas pedagógicas que adquirían, alrededor de los años mencionados, gran prestigio, por hallarse apoyada sólidamente en una nueva filosofía de la educación.

En efecto, la pedagogía alemana, de la que fue uno de sus más dignos representantes en Chile, don Jorge Enrique Schneider, había sometido a una crítica renovadora el problema fundamental de todo régimen de enseñanza: me refiero a su orientación general.

Era frecuente considerar hasta ese momento, como una verdad de carácter indiscutible, que la inteligencia del niño poseía tal ductilidad como para asimilar toda clase de conocimientos elaborados por adultos. En cambio, frente a las nuevas concepciones, el joven educado pasaba a ocupar el centro alrededor del cual la técnica debía desenvolverse.

Desde ese momento, las más interesantes investigaciones se dirigieron a obtener un concepto cada vez más claro de las modalidades propias de la vida de los escolares. Algunos psicólogos comenzaron a estudiar las características de los adolescentes.

De acuerdo con esta tendencia, una de las primeras reformas realizadas en Chile por la acción del profesorado alemán e iniciativa del Instituto Pedagógico, fue el establecimiento del plan de estudios llamado concéntrico. Al sistema de ramos separados, que exigía del alumno un gran esfuerzo de memoria, le sustituyó una prudente y gradual dosificación de materias, distribuidas a lo largo de los seis años de humanidades. 
Pues bien, la idea fundamental que presidio esta reforma consistía, precisamente, en considerar que la enseñanza secundaria debía adaptarse a las exigencias de una edad determinada, la que posee intereses que le son propios y capacidades específicas. $\mathrm{Y}$ ha sido de tal manera fértil y profunda esta doctrina, que ella se encuentra presente en el fondo de toda organización posterior, hasta sellarse con el carácter de conquista definitiva en 1935, con la declaración contenida en los programas actuales vigentes en los liceos.

Las autoridades educacionales definen los objetivos de los distintos grados de la enseñanza, fijan planes y programas, a veces también, señalan modalidades metodológicas correspondientes. Al profesorado corresponde, a su vez, poner en práctica las reformas. A este respecto, desde la fundación del Instituto Pedagógico hasta nuestros días, existe una abundante documentación que pone de manifiesto no sólo el afán del Gobierno de la República por mejorar el sistema de enseñanza, sino los propósitos de perfeccionamiento que animan al profesorado. Congresos Pedagógicos, publicaciones de obras didácticas, nuevas exigencias relacionadas con la mejor preparación del personal docente, comprueban nuestros asertos.

Sin embargo, si se desea penetrar más a fondo para comprender mejor el nuevo espíritu que anima actualmente a nuestra enseñanza secundaria, no sería suficiente ponerse en contacto con Archivos y Bibliotecas. Habría que situarse, en cambio, en el propio gabinete de experimentación que no es otro que el Liceo y la sala de clase.

En este terreno y como un homenaje de justicia a don Jorge Enrique Schneider, desearíamos demostrar cómo su influencia saludable persiste entre nosotros, a través de las tradiciones que el Liceo de Aplicación ha sabido mantener.

Schneider, su primer Rector, aunque fue un teórico de prestigio, además de sus libros y lecciones, nos ha legado una obra más valiosa y permanente: su actitud frente a los niños.

En la vida de un colegio, uno de los aspectos más interesantes es su régimen disciplinario. El Rector Schneider estimaba que un profesor no puede tener problemas de conducta si sabe interesar a los alumnos. Tomando un papel que no le correspondía, era frecuente que se preguntara ¿qué errores habré cometido que no he logrado interesar?

Es evidente que si esta convicción hubiera sido la única directiva en el señor Schneider, su pedagogía no habría rebalsado los límites de un bien entendido intelectualismo. Pero, el maestro pensaba también, en el empleo de la actividad de los alumnos en otra clase de esfuerzo. Luchando con los mayores obstáculos naturales, logró conseguir que se habilitaran unos terrenos, próximos al Liceo, en donde, poniéndose en contacto con la naturaleza, los niños pudieran dedicarse al cultivo de la tierra. Es la obra precursora de los ramos técnicos en nuestros colegios 
secundarios, que fueron más tarde establecidos para llenar una interesante finalidad de competición educativa, en armonía con la enseñanza intelectual.

Revisando las Actas del Consejo de Profesores, se puede fácilmente comprobar cómo el Rector Schneider promovía frecuentes reuniones, para deliberar en conjunto sobre problemas pedagógicos. Tomando en cuenta que los principios educativos de aquella generación se hallaban fundamentados con solidez, es explicable la actitud de los maestros que estudiaban inmediata y concretamente la mejor forma de relacionarse con la realidad chilena.

Algunas de las decisiones de esos Consejos llegaron a ser puestas en conocimiento del Supremo Gobierno y fueron adoptadas con rapidez como normas generales del servicio.

Es probable que esta labor, continuada más tarde figure como uno de los más legítimos méritos del Liceo de Aplicación.

Por otra parte, siempre aparecerá como del mayor interés el que se considere a los alumnos, simultáneamente, a través de los diversos criterios de sus maestros, para poder sacar así factores comunes de estimación y considerar los mejores estímulos para el mejoramiento de su conducta y aprovechamiento.

La colaboración entre el colegio y los padres de familia fue comprendida por Schneider en todo su verdadero alcance.

Si el hogar no prestigia la labor del profesor, si los hábitos de trabajo que se forman en la escuela son interrumpidos bruscamente en la casa, si existe una completa disparidad ideológica entre estos dos ambientes, se producirá, fatalmente, una disgregación de la personalidad del educando.

Es frecuente encontrar a muchachos absolutamente desorientados: no saben qué carrera seguir, no afirman nada con certeza, carecen de ideales constructivos.

Tales estados de ánimo obedecen, en muchas ocasiones, a las diversas solicitaciones entre la familia y el colegio.

$¿$ ¿Ha meditado el profesorado lo suficiente sobre este problema?

Continuando la obra de su primer Rector, muchas veces he sido testigo de las iniciativas que se han realizado en el Liceo de Aplicación en el sentido indicado, pero, este es un campo tan vasto e interesante que aún quedan muchos aspectos que explotar.

Por aquellos años en que Schneider observaba con profundo sentido de la ciencia universal, la escuela positiva se mantenía aún prepotente, influenciando poderosamente al rumbo de nuestra enseñanza; esta doctrina había comenzado por atacar toda tendencia dogmática, ya que su base era un racionalismo crítico. Pero como frecuentemente ocurre en la historia del pensamiento, había terminado igualmente por dogmatizar, asegurando la absoluta validez del proceso inductivo. 
Schneider alcanzó a llamar la atención acerca de las consecuencias que esta actitud filosófica podría significarle a las generaciones jóvenes.

Ha sido necesario que sobrevenga la crisis de algunas teorías científicas contemporáneas para que se dejen de considerar como indiscutibles ciertas conclusiones en las cuales se han apoyado toda una serie de proposiciones de valor moral y cultural.

También Schneider se dio cuenta del peligro que significaba el multiplicar la mera información científica, bajo el pretexto de enseñar a observar la naturaleza. Ha sido precisamente esa tendencia la que ha dado un carácter enciclopédico a ciertas asignaturas, defecto que solo ha sido posible hacer desaparecer después de grandes esfuerzos, reduciendo los programas.

En el Congreso Pedagógico de 1901, Schneider intervino activamente, expresando su opinión en algunas materias de carácter general y en otras de índole especializada.

Refiriéndose a la enseñanza de la historia de Chile demostró cómo ella, a pesar de su excepcional importancia, no se podía enseñar aislada de la cultura universal. Esta doctrina es la que ha imperado precisamente, en los programas que están actualmente en vigor, contra la opinión de los que desearían constituir con ella una asignatura enteramente aparte.

Fueron tan sólidos los principios básicos en que Schneider apoyó su sistema pedagógico que ellos podrían figurar perfectamente como la mejor justificación de nuestra manera de ser frente a los alumnos.

El niño como centro de la escuela, el interés como base del proceso del aprendizaje, la preparación para la vida, como última finalidad.

Naturalmente que estas conclusiones, como podría llamárselas, han debido ser adaptadas, incorporándoles los progresos de la ciencia moderna. Así, en la actualidad, la técnica multiplica los recursos para mantener el interés, de acuerdo con la psicología de la adolescencia y la preparación para la vida toma diversos acentos, hasta convertirse, en los tiempos que corren, en la necesidad de fortalecer el carácter a través de los conocimientos y la acción.

El ejemplo de la generación de 1900, que supo tan dignamente interpretar a su época, es el mejor estímulo para los que vivimos cuarenta años más tarde en una nueva etapa de la historia, en la que será necesario aplicar probablemente los mismos principios, pero a las más novedosas e interesantes situaciones y ésta será la filosofía del porvenir.

S.P. y L. 